\section{EROSIVE AND GANGRENOUS BALANITIS}

\section{THE FOURTH VENEREAL DISEASE}

$$
\text { B. C. CORBUS, M.D. }
$$

AND

FREDERICK G. HARRIS, M.D.

Adjunct Professor of Dermatology, College of Physicians and Surgeons, Medical Department, University of Illinois CHICAGO

Simple balano-posthitis, due to retained smegma, the secretions of gonorrhea, chancroidal infection and the decomposition of diabetic urine, has long been recognised by the various authors on genitourinary surgery. A specific form of balanitis has been recognized by French authorities for many years and more recently by German authors. The subject seems to have received but slight attention in this country. After a careful survey of the American text-books, including Watson and Cunningham, Keyes and Keyes, Fuller, Taylor, White and Martin, we are unable to find a description of a balanitis due to a specific organism.

At the medical congress in London in 1881 Simon $^{1}$ demonstrated a yeast, together with many motile spirochetes, in the secretions from the sulcus coronarius of a diabetic patient.

Bataille and Berda. ${ }^{2}$ in 1889-1891 separated and described a contagious form of balano-posthitis, which they later called balano-posthite érosive circinée, and demonstrated its contagiousness experimentally.

Ceillag and Drulle ${ }^{3}$ found a spirillum in inen which was identical with those on the clitoris of women.

In September, 1904, Scherber and Müller ${ }^{4}$ examined fifty caces of balanitis erosiva in Finger's clinic; they were able to isolate a spirillum and a vibrio and to confirm the findings of Bataille and Berdal. The same observers, stimulated by their findings in balanitis erosiva, examined six cases of gangrenous balanitis, and were able to demonstrate the same group of organisms. To those men belongs the distinction of establishing balanitis erosiva circinata and balanitis gangrenosa as a distinct malady, which they called, and, we believe, justly, a fourth venereal disease.

Recently we had under our observation five cases of this specific form of balanitis. Of these we wish to report in detail three case histories.

In presenting this subject we shall have to consider briefly the spirochetal diseases. These include:

1. Recurrent fever.

2. Frambesia tropica.

3. Dourine, a venereal disease of horses.

4. Syphilis.

5. Vincent's angina and other ulcerative diseases of the mouth.

6. Gangrenous processes occurring in various parts of the body, and including the disease under discussion.

The first three diseases, recurrent fever, dourine and frambesia tropica, are of interest to us only inasmuch as they are each due to a specific form of spirochete. Syphilis is now conceded by all to be due to the Spirochceta pallida, whose morphology has been so well described as to need mention only.

The spirochetes found in Vincent's angina, ulcerative stomatitis, noma and other gangrenous processes are so

1. Simon, O.: Balano-posthomykosis, Tr. Internat. Med. Cong., London, 1881, ili, 138 .

2. La balano-posthite érosive circinée, Méd. moderae, 1891, 1i. 340 .

3. Quoted by

4. Arch. f. Dernat. u. Syph., Ixxvil, 77. closely associated with balanitis erosiva or gangrenosa as to merit special discussion.

It has long been known that several forms of spirochete were normally found in the mouth. The following, on account of their intimate relation to our subject, will bear a somewhat detailed description:

1. The Spirochata buccalis of Cohen.-This organism is 10 to $20 \mathrm{~mm}$. long by $0.5 \mathrm{~mm}$. wide. It often appears threadlike or resembling a whip; the windings are long. the ends are sharp and end in the center of the spiral; they may be ciliated. In the hanging drop they are very refractile and show a bending and snakelike motion. They are very active and vary greatly in size and thickness. In comparison with other forms of the spirochete they are characterized by their greater width. In the body are found at times red nuclear granules.

2. The Spirochata dentium of Koch.-This spirochete is the smallest and most delicately formed. It usually measures about 4 to $10 \mathrm{~mm}$. in length and is so thin that the breadth is not measurable. Its windings are short and regular. Its elasticity is marked.

This form simulates the Spirochata pallida somewhat closely. Whether or not these two forms represent varieties of one and the same organism has not been demonstrated.

These organisms have the following features in common:

1. Drying easily kills them.

2. They stain in the cold with the ordinary stains.

3. They are Gram-negative.

4. With the Giemsa stain they are colored bluish-red.

5. They are anaerobic.

Of the spirochetal diseases that occur in the mouth we have Vincent's angina, noma and mercurial stomatitis. In all these conditions the exciting cause seems to be the same.

In Vincent's angina the etiologic factors are a vibrio and a spirochete. This was confirmed by Rauchfus and Plaut in 1893-4. The primary location is usually on the tonsil and edge of the gums and is characterized by the formation of a pseudomembrane which is easily removed, disclosing ulcers. These may become confluent; sometimes they are very superficial. The submaxillary and retromaxillary glands are enlarged, firm. but rarely suppurate. The constitutional symptoms are slight.

Following the exanthematous disease, particularly measles and diphtheria, there occurs at times a gangrenous stomatitis or noma, characterized by extensive gangrene of the cheek, which may extend to the maxillary bones, in most cases being followed by death.

The first symptom to attract attention as a rule is the offensive odor of the breath. On examination a black necrotic spot is seen on the inner surface of the lip or cheek, surrounded by tissues that are intensely swollen and edematous. This rapidly becomes in turn gangrenous and we may have extensive sloughing of the tissues. In the border of the necrotic membrane we find the fusiform bacillus and the spirochete in pure caltures.

Matzenauer ${ }^{5}$ asserts that noma and hospital gangrene are due to the same cause, which is identical with the organism found in Vincent's angina.

5. Matzenauer, R. : Noma und Nosocomialgangrän, Arch. f. Dermat., 1902, 1x, 373; ibid., 1901, 1v, 67. 
Rona ${ }^{6}$ in 1905 examined three cases of noma following measles. His conclusions are as follows:

Noma begins without exception in gangrenous stomatitis. If the fusiform bacillus and spirochæte found in the mouth are etiologic factors in gangrenous stomatitis, since the organisms are found in such abundance in noma, it must be due to the same cause.

Since all these conditions are favored by lowered resistance, which is produced by the exanthematous diseases and the administration of mercury, it is not to be wondered that organisms that are saprophytic can easily become pathogenic.

Lichtwitz, ${ }^{7}$ Sabrazis $^{7}$ and Silberschmidt ${ }^{8}$ found the organism in the antrum of Highmore, Miller ${ }^{9}$ in an alveolar abscess, Pollard ${ }^{10}$ in an abscess of the leg.

Perthes, ${ }^{11}$ Krahn, ${ }^{12}$ Ellerman ${ }^{13}$ and Buday ${ }^{14}$ fcund the organisms in the deeper layers of the tissues; Scherber and Müller ${ }^{4}$ found them in the lumen of the vessels, whence they may disseminate and produce a spirochetal septicemia or cause metastatic abscesses. Plautt ${ }^{15}$ found the organism in a tonsillar abscess, Raoult and 'Thiery' in a subtonsillar abscess.

Schmor ${ }^{16}$ demonstrated the organism in abscesses of the liver, spleen and lung, and other observers have found them in abscesses of the brain and appendiceal stump; so we see that there is an abundance of clinical evidence showing the pathogenicity of the organism.

In the preputial sack the moisture and warmth favors the growth and, as these organisms are anaerobic, this location is an ideal culture ground.

\section{DEFINITION}

Erosive or gangrenous balanitis is a specific infectious venereal disease, caused by a symbiosis of a vibrio and a spirochete, with local and constitutional symptoms varying with the severity of the infection.

\section{ETIOLOGY}

The first predisposing cause is a long tight foreskin, which seems to exclude the air. This must always be present in a greater or less degree, as the organisms which produce this form of balanitis are anaerobic. The second is wetting the labia or penis with saliva. As thts form of balanitis is so closely associated with noma, Vincent's angina and mercurial stomatitis in its bacteriology, it is highly probable that the cause of the infection is the same in the two diseases. The third is unnatural sexual relations due to alcoholism.

The existing cause of this form of balanitis, as stated before, is a symbiosis of a vibrio and a spirochete. These two organisms are always found together, but, as the spirochetes are found under normal conditions in the preputial sac, it is highly probable that the pathogenic. factor is the vibrio. This organism occurs most abundantly in the deeper layers of the necrotic membrane, while the spirochetes are found less abundantly and in the upper portion of the necrotic membrane. Both have been demonstrated in sections, in the blood vessels and in the inguinal glands.

The vibrio grows under anaerobic conditions on serum agar. It occurs singly or in chains of two or

\footnotetext{
6. Arch. f. Der ma: u. Syph., lxxiv, 171.
7. Quoted by Feldmann: Wien. klin. Wchnschr., 1906, p. 696.

7. Quoted by Feldmann: Wien. klin. Wchn

9. Deutsch. med. Wchnschr., 1906, No. 9, 348

9. Deutsch. med. Wehnschr., 1906, No. 9, 348 . Wien. klin. W chnschr., 1905, xvili, 1236 .
10.

11. Wünchen, med. Wchnschr. 1902, xlix, 1968 .

11. München. med. Wchnschr., 1902, xlix, 1968.

12. Mitt. a. d. Grenzgob. d. Med. u. Chir
13. Centralbl. f. Bacteriol., xxxviii, 383 .

14. Beltr. z. patb. Anat. u. z. allgem. Path., 1905, xx上viii, 255.

15. München. med. Wchnschr., 1907, liv, 340.

16. Müchen. med. Wchnschr., 1907, No. 4, p. 188.
}

more individuals. It is a slightly curved rod-shaped organism with pointed ends, measuring from $2 \mathrm{~mm}$. in length to $0.8 \mathrm{~mm}$. in width. It stains by the ordinary dyes and is Gram-positive, although the decolorization must be performed very carefully, as the organism gives up the gentian violet very readily. It is preferable to use 70 per cent. alcohol for this purpose.

The spirochete is Gram-negative, but stains with the ordinary dyes; with the Giemsa stain it takes a bluish red. These organisms are best seen with the dark ground illuminator. They average from 6 to $30 \mathrm{~mm}$. in length and about $0.2 \mathrm{~mm}$. in width. The windings are not acute and the ends of the organism terminate in the center of the spiral. The motion of the organisms is very rapid; they travel from place to place, resembling a small snake; they have a rotary motion, but this is not as pronounced as the backward and forward motion.

\section{PATHOLOGY}

The pathologic condition in the milder forms of balanitis erosiva circinata consists simply of a flaking off of the epithelium, leaving small superficial erosions. When the desquamation is more marked there are bright red ulcers, which are surrounded by a small white zone, the remains of the necrotic epithelium.

In the surrounding tissue there is an exudation of leucocytes and plasma. The organisms are found in the secretions from the erosions, but more abundantly in the necrotic membrane. At times they can be demonstrated in the lissues and blood vessels, as shown by Scherber and Müller.

In the more severe grades of infection there is more venous stasis and more exudation, resulting in marked phimosis which predisposes to gangrene. As Scherber and Müller pointed out, the whole condition is one of degree only, but for clinical purposes we may distinguish two types: (1) Balanitis erosiva circinata and (2) balanitis gangrenosa.

\section{SYMPTOMS}

Balanitis erosiva circinata commences with the appearance of one or more small grayish white patches in the preputial sack. At the time of the development of the erosion an offensive thin pus is produced, with a characteristic stinking odor and of the usual vellowishwhite color; in the more severe cases it becomes grayishwhite or grayish-brown.

In the mild cases the foreskin may be easily retracted, but in the more severe forms marked phimosis develops; there is considerable itching and burning behind the glans; the act of urination is practically without pain. In contradistinction to the gangrenous form in this type of the disease, constitutional symptoms are slight or absent.

Generally the sulcus coronarius is the site of predilection; next, the adjacent part of the prepuce, and, last, the glans.

As the process follows no hard and fast lines there are certain deviations from the above picture. The process may be limited to the glans and the inner surface of the foreskin be unaffected. This may be extreme or mild, but is always present on the covered portions of the glans.

The inflammatory condition may remain a purely erosive superficial process and may recover spontaneously, or by tize aid of mechanical cleansing and antiseptic treatmerit. 
In a number of cases the process does not remain superficial, but develops deep diphtheritic and gangrenous ulcers, which complicate the clinical picture in many ways.

In some cases when one is able to retract the foreskin, one sees after removal of the pus, inside of the erosions small round ulcers varying in size from a pin-head to that of a pea.

These ulcers are moderately deep and on the whole flat and surrounded by a red zone. They are covered by a closely adherent pseudomembrane. İn other cases the ulcers are more extensive and deeper, the average size being about that of a dime. These may become confluent and extend over the whole surface of the sulcus or the inner surface of the foreskin.

These balanitic ulcers are of a somewhat irregular outline and are surrounded by a small inflammatory slightly elevated border. This border is clean-cut and the sides somewhat slanting; the base is uneven with a firm yellowish-white or yellowish-brown membrane, which is often edematous and swollen. When more edematous this false membrane appears as a sort of friable slime. Here and there may be hemorrhagic spots which sometimes give rise to hemorrhages from the base of the ulcer.

In the severe forms the constitutional symptoms are more marked. Scherber and Müller noticed in a majority of their cases chills and fever and at the onset vomiting, the average temperature ranges from 100 to 101 degrees. There is marked edema, the external skin being red and edematous; the infiltration may extend to the root of the penis in some cases. The dorsal lymph cord is usually palpable and the inguinal glands are enlarged, but not painful. Unless the phimosis is complete there is no pain on urination; when, however, the urine is not able to pass freely and dilates the preputial sac there is considerable pain.

The discharge is the most profuse in this type of the disease. By gently irrigating the preputial sac with sterile water and wiping the external urethral orifice we can easily exclude a gonorrhea by having the patient urinate in two glasses.

In the majority of cases of balanitis gangrenosa there occurs a marked edema of the subcutaneous tissue of the penis which extends to the root and causes a marked phimosis. If the ulcer is situated on the inner surface of the foreskin it shows externally as a dark, bluish-red area within the surrounding bright red inflammatory tissue. The congestion and abnormal pressure, due to the edema, favor in a marked degree the progress of the disease.

Soon the foreskin over the ulcer becomes black and a complete necrosis of the part occurs. If the ulcer is situated on the glans, in a short time it may produce complete destruction of the same or may even cause an extremely rapid gangrene of the organ which may extend even to the root of the penis, as may be seen by the third case reported in this paper.

The ulcers in these cases are deep, the edges sharp and perpendicular, the base grayish-green or brownish; or it may show hemorrhagic areas or be changed into a black necrotic mass.

The discharge at this time is more offensive than in the erosive type; it has a grayish-yellow or grayish-brown color and at times it may be slightly hemorrhagic, but always with the same characteristic odor. The inguinal glands are enlarged; there is a mild grade of sepsis present. General malaise is marked. There may be vomiting and the temperature may reach 104 . The tenderness of the part is extreme.

\section{DIAGNOSIS}

This disease is not so uncommon as one might suspect; in fact we believe that it is very common and is usually mistaken for chancroidal infection. The period of incubation may be the same in the two conditions; but with the characteristic thin yellowish white offensive discharge, in which one finds a vibrio-form organism and a spirochete, the diagnosis should not be difficult.

The ulcers of the two forms of infection may simulate each other very closely. In this form of balanitis when the infection is at all severe there is marked phimosis and considerably more inflammatory reaction. The enlarged inguinal glands are painless, while with a very insignificant chancroidal sore a suppurating bubo is often present.

Chancroidal ulcers are as a rule multiple, but they do not spread with as great rapidity as do the ulcerative forms of balanitis. Whereas the ulcers in both diseases have a clean-cut punched-out appearance, there is greater tendency to undermine the wall in chancroidal infection.

In chancroidal infection we find the Ducrey-Unna bacillus and do not find the vibrio or the spirochete.

On account of the indolent adenopathy that accompanies balanitis erosiva, it must be differentiated from syphilis. In syphilis the period of incubation is longer, although the two infections may occur simultaneously, as reported in one of Scherber's cases. When such a condition exists we may be compelled to defer our diagnosis of syphilis until the period of incubation for syphilis has elapsed; or in case of a mixed lesion the Spirochceta pallida may be easily demonstrated by the dark ground illuminator and is so characteristic as to be easily differentiated from the spirochete of balanitis.

Herpes preputialis always occurs as groups of small insignificant vesicles in which local reaction is mild or entirely absent. This condition simulates somewhat the mild form of balanitis erosiva, but in herpes one fails to find the organisms characteristic of balanitis.

\section{TREATMENT}

As a prophylactic measure the practice of circumcision should be encouraged; it is absolutely impossible for balanitis to exist in an individual who has been circumcised.

In many cases in which the condition is mild and the foreskin can easily be retracted all that is necessary is a thorough cleansing, but in the mild ulcerative forms in which there is the slightest evidence of phimosis a dorsal incision should be performed. As the organism of balanitis is anaerobic, this incision serves a twofold purpose, that is, of admitting air and exposing the diseased parts for treatment.

Previously we were in the habit of burning all these sloughing ulcers in this disease, but such treatment subjects the patient to needless punishment. As we have said above, the organisms of the disease are anaerobic, and as hydrogen peroxid liberates oxygen when in contact with organic matter, it acts as a specific for this form of infection.

We use the ordinary 2 per cent. solution, but in severe cases of gangrenous balanitis, 25 per cent. was painted on the parts. 


\section{REPORT OF CASES}

CASE 1:-History.-The patient, M. M. W., aged 40, married, denied all previous venereal history. After four days' incubation the patient noticed itching and burning around the glans penis. There were no constitutional symptoms. During the first week this continued as a mild balanitis. The patient was able to retract the foreskin. Treatment was neglected. At the end of the first week conditions suddenly became worse; the foreskin began to swell and the patient was unable to retract it. At this time he presented himself for examination.

Examination.-The general muscular development was good; there were no scars or evidence of previous venereal disease. The penis was swollen and edematous; the edema extended about half way up the shaft of the penis, giving it a pea: shape. The skin over the glans portion was red and slightly injected. There was complete phimosis. Exuding from the opening was thin, yellowish-white pus, with a penetrating odor, in which was found a vibrio and a spirochete. There was constant burning pain "which was increased on the slightest pressure. There was no urinary pain. The dorsal lymph cord was easily palpabıe; the inguinal glands were slightly enlarged but not tender. There was no fever.

Treatment.-With a small hand syringe 2 per cent. hydrogen peroxid was injected every hour into the preputial sack. By the second day the foreskin could be retracted, showing numerous small ulcers with sloughing bases with sharp borders, involving the sulcus and the covered portion of the glans.

These healed rapidly under the above treatment.

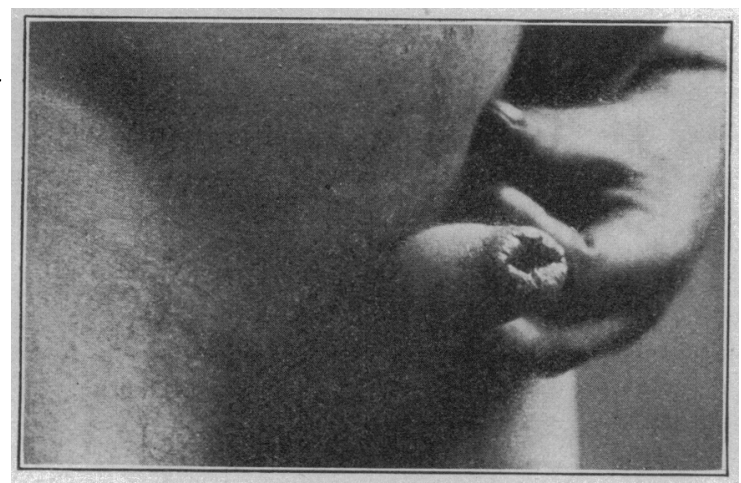

Balanitis erosiva seu gangrenosa, erosive type.

CASE 2.-History.-The patient, M. W. M., aged 26, Iris'n, denied syphıis: had had a supposed chancroidal infection two years previously. Two weeks before presenting himself, the patient had intercourse. After three or four days there was a little itching beneath the prepuce. At the end of six days he presented himseif for examination.

Examination.-The temperature and pulse were normal. The general nutrition was good, and there were no signs of latent syphilis. There was a large indurated swelling of the penis. From the preputial orifice exuded a thin, yellowish-white, stinking discharge. This was examined for gonococei but none was present. There was phimosis, but not complete. With dilatation, the little finger was gently passed between the foreskin and the glans. The whole covered portion of the glans and the inner leaf of the foreskin was covered with small ulcers, having necrotic, sloughing bases. Those on the inner leaf extended to the border of the preputial fold; by gently pulling back the foreskin the whole could be plainly seen The dorsal lymph cord could be plainly felt and the inguinal glands were enlarged but not tender. There were no constitutional symptoms.

Treatment.-The patient was given a wash of hydrogen peroxid, full strength. As he did not return to the clinic, we presume that his condition was satisfactory.

Case 3.-History.-The patient, A. G. G., aged 43, American, denies all previous venereal history. He had had intercourse nine days firevious; at this time, the patient said the prostitute lubricated her labia with saliva. The following day the glans portion began to swell; there were chilly sensations, no nausea or vomiting. Previous to this time the patient's glans penis was exposed between the preputial fold and the foreskin could be retracted. On account of the rapid phimosis that developed this could not be accomplished later. The local symptoms increased rapidly; by the third day gangrene had set in.

Examination.-When the patient presented himself at the clinie he was well nourished; muscular development good. There was slight septic intoxication. The entire preputial covering for a distance of three inches was one black, necrotic mass. By gentle manipulation the necrotic mass could be drawn away and deep sloughing ulcers, with sharp borders, could be seen extending into the penis above the glans. There was considerable thin, slimy pus, with an odor of necrotic tissue present. Here we were able to find the organism in large numbers. The remaining portion of the penis was dark red and infiltrated the edema extending to the root; the inguinal glands were enlarged. The patient's temperature was 102 ; malaise was marked.

Treatment.-The patient was sent to the county hospital. Here the necrotic foreskin was cut away, and just above the glans portion, at the site of the inner preputial fold, could be seen two deep ulcers. The glans portion was necrotic. In forty-eight hours the entire glans portion, together with about one and a half inches of the shaft of the penis, sloughed off, leaving a short stump. The patient was treated with irrigations of potassium permanganate three times a day, but the organisms had already invaded the deeper layers and gangrene was unavoidable.

\section{CONCLCSIONS}

1. We agree with Scherber and Müller that there is a characteristic clinical picture or form of erosive gangrenous balanitis, which under favorable conditions may cause deep and widespread gangrene, and which is of sufficient importance as to be called a fourth venereal disease.

2. In the erosice and gangrenous types there is regularly found a micro-organism (vibrio), which we consider the cause of the disease. In addition to this organism there is constantly present a Gram-negative spirochete.

3. On account of the rapid development of phimosis with a tendency to produce gangrene, it is inperative that a diagnosis should be made at once.

4. As we have a specific in hydrogen peroxid, its early use will prevent extensive destruction.

5. Whether these organisms are identical with those found in similar processes in the mouth and pharynx is still a question.

103 Randolph Street.

\section{CERVICAL DILATATION}

A SIMPLE (AND ORIGINAL?) METHOD WITHOUT LOCAL OR GENERAL ANESTHESIA *

CURRAN POPE, M.D.

Professor of Physiotherapy in the University of Louisville, Medical Department LOUISVILLE, KY.

Dilatation of the cervical canal of the uterus does not yet stand on a thoroughly scientific basis; nor is its mode of action in some cases clear. Probably a mechanical or neuromechanical hypothesis would come nearer answering in most instances, than any other explanation. We all know that its results have not by any means been uniform; sometimes they have been good, oftentimes poor and in other instances absolute failures. Considerable observation has led me to believe

* Read before tije Louisville Society of Medicine. 